\title{
First detection and molecular characterization of kobuvirus from a dog in Turkey
}

\author{
ZEYNEP AKKUTAY-YOLDAR, B. TAYLAN KOÇ*, T. ÇIĞDEM OĞUZOĞLU
}

\begin{abstract}
Department of Virology, Faculty of Veterinary Medicine, Ankara University, Diskapi 06110, Ankara, Turkey *Department of Virology, Faculty of Veterinary Medicine, Aydın Adnan Menderes University, 09016, Aydın, Turkey
\end{abstract}

Akkutay-Yoldar Z., Koç B. T., Oğuzoğlu T. Ç.

\section{First detection and molecular characterization of kobuvirus from a dog in Turkey}

\section{Summary}

Canine kobuvirus (CaKVs) is a newly emerging virus detected in dogs in several countries. However, kobuvirus infection has not yet been described in domestic carnivores in Turkey. In this study, we tested blood and rectal swab samples to determine the presence of kobuvirus in a dog with clinical symptoms by reverse transcription-polymerase chain reaction (RT-PCR), using 3D (RNA polymerase) region primers of canine kobuviruses. To provide molecular characterization data, the Maximum Likelihood (ML) method was used for the phylogenetic analyses. The PCR product of the partial protein-coding region of the 3D protein gene from the rectal swab was amplified, purified, and sequenced. Phylogenetic analysis of amino acid sequences suggests that our CaKV strain was closely related to US-CaKVs, and placed on a monophyletic clade as a sister branch localized in the CaKV cluster. These results indicate that CaKV exists in dogs in Turkey. With a similarity of 94.2-96.1\%, it is like other CaKVs. To our knowledge, this is the first report of CaKV infection of a dog by in Turkey. Further studies are needed to determine its role in dog gastrointestinal infections.

Keywords: Canine kobuvirus, dog, PCR, phylogenetic analysis

Kobuvirus is a single-stranded RNA virus of 30 $\mathrm{nm}$, classified as a member of the Picornaviridae family, which is divided into 40 genera according to the International Committee on Taxonomy of Viruses (ICTV, 2017). The kobuvirus genus comprises six species: Aichivirus A, Aichivirus $B$ (bovine kobuvirus), Aichivirus $C$ (porcine kobuvirus), Aichivirus $D$ (kagovirus 1 and 2), Aichivirus $E$ (rabbit picornavirus), and Aichivirus F (bat kobuvirus) (1). Aichivirus A consists of four types: aichivirus 1 , canine kobuvirus 1 , feline kobuvirus 1, and murine kobuvirus $(6,8,14)$.

Kobuvirus genomes vary between 8.2 and $8.4 \mathrm{~kb}$ in length, with a typical picornavirus configuration. At 240 nucleotides (nt), the 3'-UTR region of aichivirus (AiV) is longer than those of Kobuvirus $B$ and $C$, at 177 and $170 \mathrm{nt}$, respectively (16). The genome has one open reading frame (ORF) encoding a single polyprotein of 7,467 nt. The capsid morphology is dissimilar from other viruses in the Picornaviridae family. There are three structural viral proteins, VP0, VP1, and VP3, encoded by the $\mathrm{P} 1$ region. $\mathrm{P} 2$ and $\mathrm{P} 3$ encode seven non-structural proteins (2A-2B-2C/3A-3B-3C-3D) $(4,11)$. From these non-structural proteins, $3 \mathrm{D}$ encodes a viral RNA-dependent RNA polymerase (RdRp). This gene region is also a preserved area for kobuviruses $(1,16)$.

Kobuviruses have been found in various animal species, including cattle, swine, sheep, goats, dogs, cats, rabbits, and birds $(9,14)$ and were classified into a new genus, Kobuvirus, in 1999 (4). The prototype kobuvirus (human aichivirus), obtained in 1989 in Aichi province, Japan, was identified eight years later. Its first detection in cattle was again in Japan in 2003 $(19,20)$. Porcine kobuvirus, which is common in piglets, was first detected in pigs without any clinical symptoms in Hungary in 2008 (16). Kobuvirus was first detected in dogs from gastroenteritis cases in 2011 in the United States. Sequence analysis indicates that all these viruses were canine kobuviruses (CaKV) (7). Meanwhile, CaKV detected from three diarrheic dogs was classified as a new species related to $\mathrm{AiV}$ in the genus Kobuvirus (10).

While CaKV infection in dogs has been reported from several different countries, no study has been carried out in Turkey. We therefore aimed to determine whether CaKV infection is present in Turkey, and to demonstrate its relationship with other kobuviruses in other countries. In this study, we report the first 
detection of $\mathrm{CaKV}$ in a rectal swab of a diarrheic dog in Turkey.

\section{Material and methods}

Samples. Blood and rectal swab samples from a dog with clinical signs, specifically diarrhea, fever, and vomiting, were taken into EDTA blood tubes in May 2018 in Ankara, Turkey. The specimens were placed in ice bags and transferred to the lab, where they were kept frozen at $-80^{\circ} \mathrm{C}$ until tested.

Polymerase Chain Reaction (PCR) and phylogenetic analysis. The blood and swab samples were used for total RNA extraction according to the phenol: chloroform: isoamylalcohol extraction method described by Chomczynski and Sacchi (3). cDNA synthesis carried out with MMLV-RT using random primers (Fermentas, Lithuania) isolated $3 \mu \mathrm{l}$ of RNA. The RNA pellets were resuspended in $20 \mu \mathrm{l}$ of deionized water and used as a template for the RT-PCR applications. All RNA was stored at $-20^{\circ} \mathrm{C}$ until testing. PCR for the partial gene was performed using kobuvirus oligonucleotide $\mathrm{CaKV} / \mathrm{F}$ sense and $\mathrm{CaKV} / \mathrm{R}$ anti-sense primers, as described by Di Martino et al. (5). The RT-PCR conditions were 40 cycles of DNA denaturation at $94^{\circ} \mathrm{C}$ for $30 \mathrm{~s}$, primer annealing at $48^{\circ} \mathrm{C}$ for $1 \mathrm{~min}$, and amplification at $72^{\circ} \mathrm{C}$ for $1 \mathrm{~min}$. The reaction tubes were then kept at $72^{\circ} \mathrm{C}$ for a further $10 \mathrm{~min}$ for final extensions. The reaction mixture contained (5 U/ $\mu 1)$ Taq DNA polymerase (MBI, Fermentas, Waltham, MA, USA), $10 \times$ Taq Buffer (1.25 ml including $\left(\mathrm{NH}_{4}\right)_{2} \mathrm{SO}_{4}$, $25 \mathrm{mmol} / \mathrm{L} \mathrm{MgCl}_{2}$, and water, $18 \mathrm{Mohm} / \mathrm{cm}$, Applichem, Darmstadt, Germany), primers, and $3 \mu 1$ of extracted cDNA in a reaction mixture volume of $30 \mu \mathrm{l}$ of mix. PCR products were visualized in a transilluminator after electrophoresis in $1 \%$ agarose gel containing ethidium bromide.

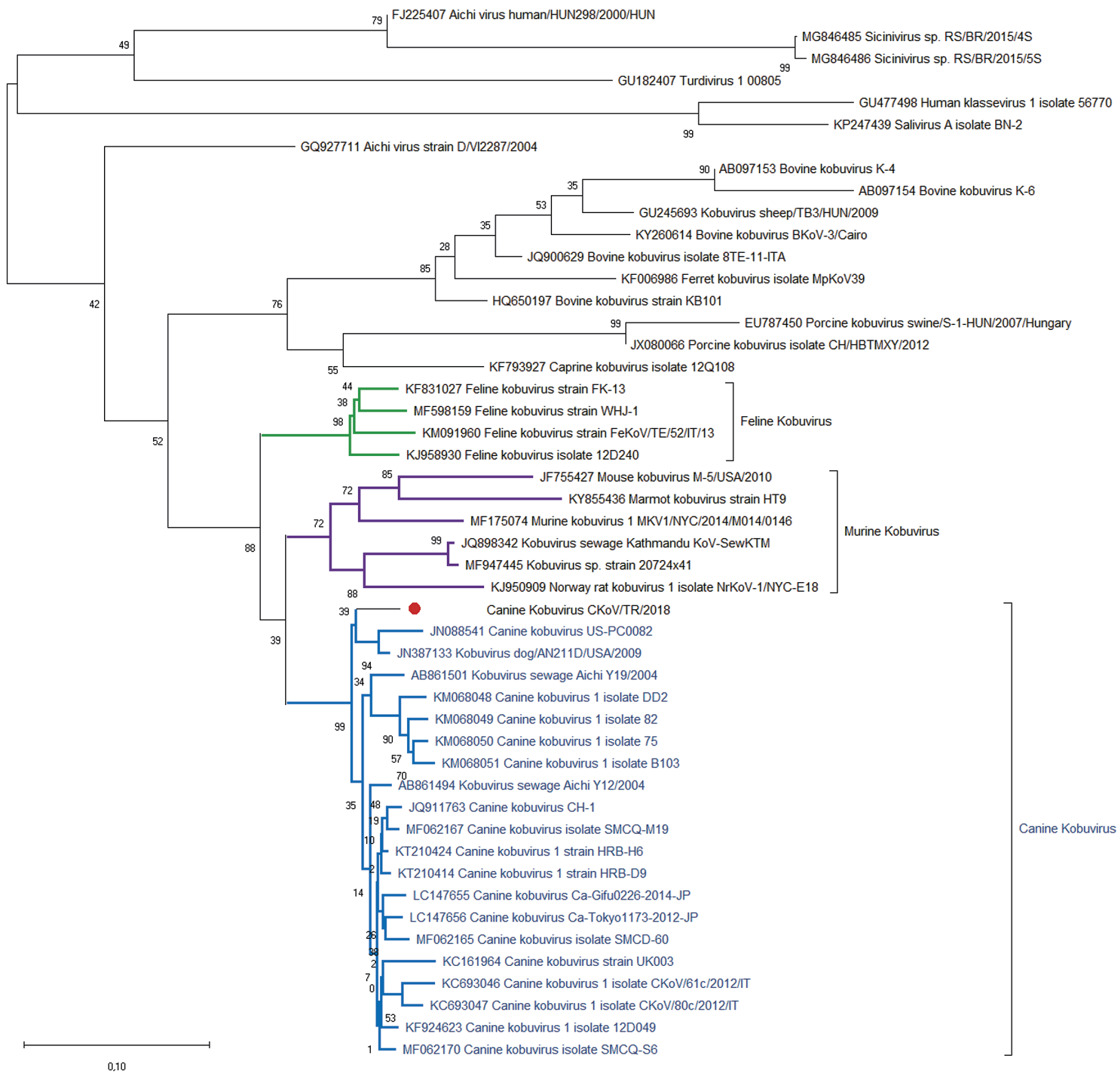

Fig. 1. Molecular phylogenetic analysis of partial 3D gene by Maximum Likelihood method 
The rectal swab PCR product was purified with a Gene JET PCR purification Kit (Thermo Scientific, Waltham, MA, USA) before the PCR fragments were sent to BM Labosis (Ankara, Turkey) for Sanger sequencing. Bidirectional sequence reading was performed to reduce errors that may occur on both sides for characterizing amplicons. Sequences from different geographical regions were taken from the GenBank for phylogenetic analysis of the partial 3D gene region of canine, feline, mouse, marmot, bovine, pig, ferret, sheep kobuviruses, and human aichivirus, klassevirus, gray-backed thrush turdivirus, and chicken sicinivirus. The obtained sequences were aligned using CLC Main Workbench v5.5 (CLCBio, Aarhus, Denmark) before analysis with MEGA 6.06 (18). Maximum-likelihood trees were generated based on the Kimura two-parameter model with 1,000 bootstrap replicates (Fig. 1). The sequence of the $3 \mathrm{D}$ (RNA polymerase) gene was submitted to the NCBI GenBank database. The nucleotide sequence described here was deposited in GenBank under accession number MK089456.

\section{Results and discussion}

The rectal swab sample screened by PCR assay using $\mathrm{CaKV}$-specific $\mathrm{CaKV} / \mathrm{F}$ sense and $\mathrm{CaKV} / \mathrm{R}$ antisense primers yielded a specific amplicon of a 504-bp product. BLAST searches revealed the sequence of the rectal swab to be $94-99 \%$ identical to various CaKV strains. A phylogenetic tree was constructed based on the nucleotide sequence alignments of the 3D gene. Analysis of the nucleotide sequence of the field virus with several kobuvirus sequences revealed that the obtained sequence grouped in the Canine Kobuvirus cluster within the USA strain group in a distinct branch with $94.5 \%$ and $96.1 \%$ nucleotide similarity to PC0082/ JN088541/CaKV/USA and AN211D/JN387133/CaKV/ USA strains respectively (Fig. 1). This result was confirmed by SDT, a stand-alone program with a graphical interface that shows pairwise identity scores using a col-
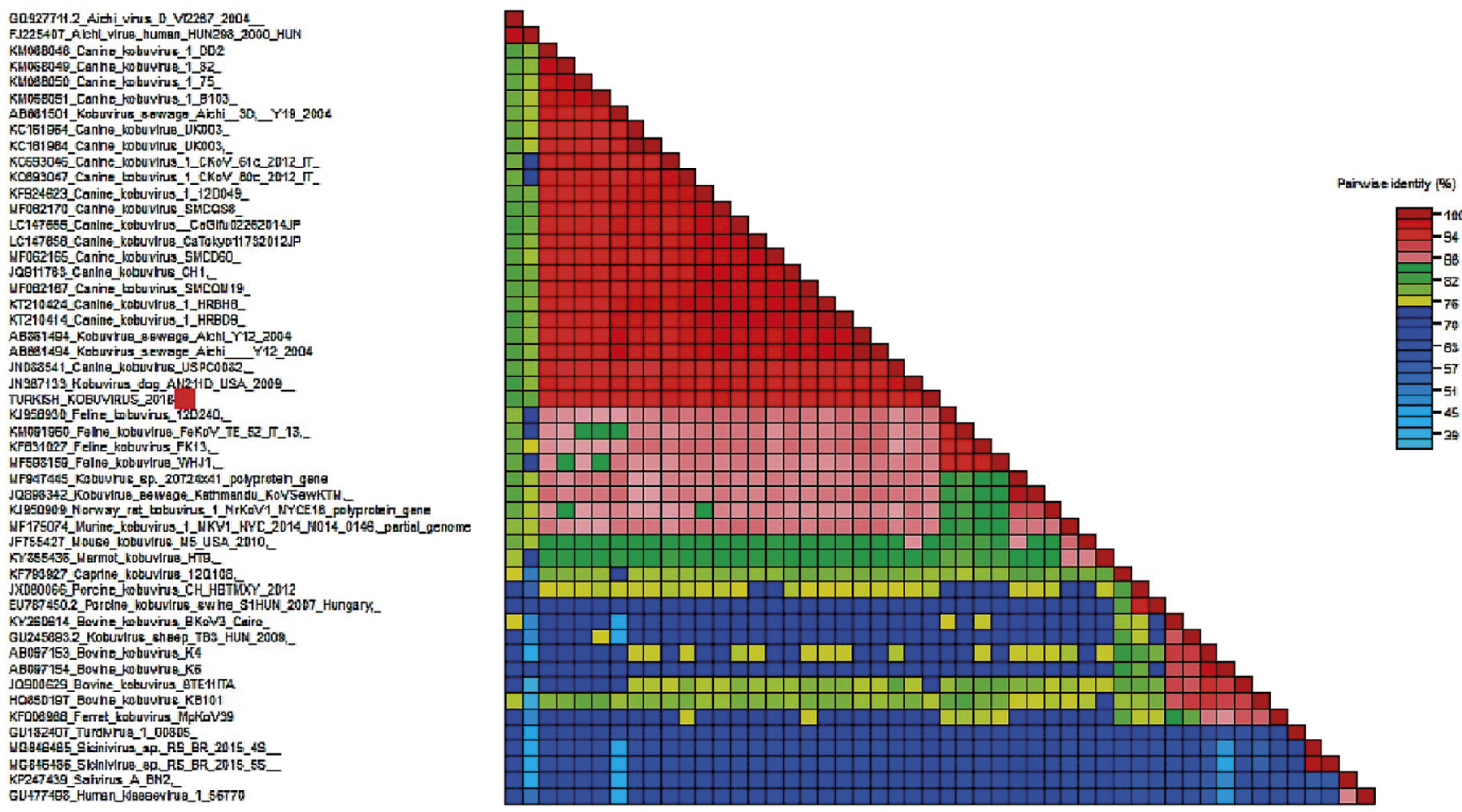

HFEg:158 Falne

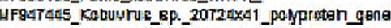

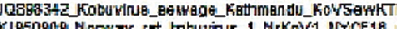

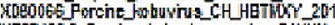

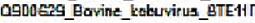

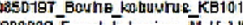

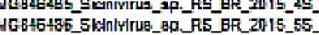

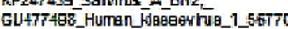
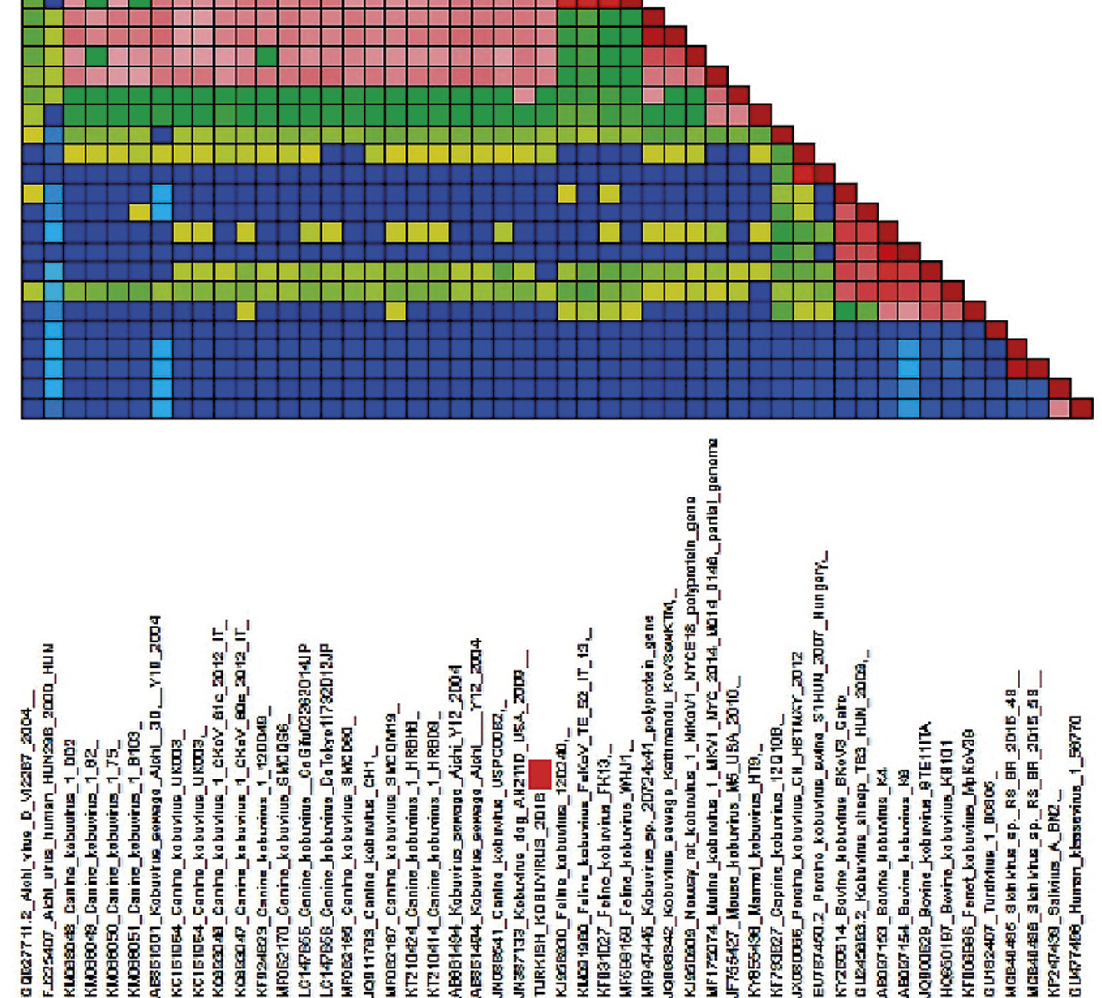

Fig. 2. SDT color-coded matrix of pairwise identity scores generated by alignment of a 480 bp fragment 3D gene for 48 viruses. Each colored square symbolizes a percentage identity score between two sequences (one indicated horizontally to the left and the other vertically at the bottom) 
or-coded pairwise identity matrix, which enables the comprehensive relationships between sequences in a dataset to be viewed (11). Applying the SDT program with pairwise deletion of gaps determined the identity matrices of 48 virus, as shown in Fig. 3. The identities decreased from $100 \%$ to $70 \%$ while the color changed from dark red to dark blue. CaKVs mostly shared a red color with other counterparts, indicating a higher pairwise sequence identity than those from feline and mouse, which fell in

\begin{tabular}{|c|c|c|c|c|c|c|c|c|c|c|c|c|c|c|c|c|c|c|c|c|c|c|c|}
\hline & & 2 & 3 & 4 & & 6 & & 8 & 9 & 10 & 11 & 12 & 13 & 14 & 15 & 16 & 17 & 18 & 19 & 20 & 21 & 22 & 23 \\
\hline CH-1 1009 & & 3 & 97.5 & 98.6 & 97.59 & 97.29 & 95.6 & 97.5 & 95.6 & 92.8 & 94.8 & 94.8 & 94.2 & 94.5 & 98.65 & 97.58 & 88.48 & 86.28 & 86.2 & 86.2 & 85.4 & 82.4 & 82.9 \\
\hline & 98.3 & & 98.1 & 99.2 & 98.19 & 97.2 & 96.7 & 98.6 & 96.1 & 93.4 & 95.3 & 94.8 & 94.2 & 95.0 & 99.2 & 98.1 & 87.38 & 86.08 & 86.5 & 86.8 & 85.1 & 83.2 & 83.2 \\
\hline $12 / 212$ & 97.5 & 98.1 & & 98.3 & 97.29 & 97.0 & 95.3 & 7.2 & 95.0 & 92.8 & 95.0 & 94.5 & 93.9 & 94.8 & 97.2 & 0.08 & 88.78 & 87.38 & 87.3 & 87.3 & 86.0 & 3.5 & 82.9 \\
\hline$A R B=C$ & 98.6 & 99.2 & 98.3 & & 98.39 & 98.19 & 95.9 & 98.3 & 95.9 & 93.7 & 95.0 & 94.5 & 93.9 & 94.8 & 98.39 & 98.38 & 88.28 & 86.28 & 88 & 86.5 & 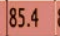 & 4 & 82.4 \\
\hline & 5 & 98.1 & 97.2 & 98.3 & & 98.1 & 95.9 & 97.8 & 95.0 & 93.9 & 95.3 & 94.8 & 93.7 & 94.5 & 97.2 & 97.28 & 88.28 & 86.58 & 86.2 & 87.1 & 85.1 & 82.6 & 82.6 \\
\hline & 97.2 & 97.2 & 97.0 & 98.1 & 98.1 & & 95.6 & 97.5 & 95.9 & 93.7 & 95.0 & 94.5 & 93.4 & 94.8 & 97.05 & 97.0 & 89.0 & 87.1 & 86.2 & 87.3 & 85.7 & 82.9 & 81.8 \\
\hline & 95.6 & 96.7 & 95.3 & 95.9 & 95.9 & 95.6 & & 96.4 & 94.2 & 92.0 & 93.9 & 93.4 & 92.8 & 94.2 & 95.99 & 95.38 & 87.3 & 86.0 & 84.6 & 87.1 & 84.8 & 3.7 & 83.7 \\
\hline & 97.5 & 98.6 & 97.2 & 98.3 & 97.8 & 97.5 & 96.4 & & 96.1 & 92.8 & 94.8 & 94.2 & 93.7 & 95.0 & 97.8 & 97.2 & 88.2 & 86.8 & 86.2 & 86.8 & 84.3 & 2.9 & 11.8 \\
\hline Kov & 95.6 & 96.1 & 95.0 & 95.9 & 95.0 & 95.9 & 94.2 & 96.1 & & 93.9 & 95.3 & 94.8 & 94.5 & 95.9 & 95.99 & 95.08 & 87.9 & 86.0 & 86.5 & 86.0 & 85.1 & 83.2 & 81.8 \\
\hline & 92.8 & 93.4 & 92.8 & 93.7 & 93.9 & 93.7 & 92.0 & 92.8 & 93.9 & & 0 & 94.2 & 92.3 & 94.5 & 93.19 & 92.8 & 86.5 & 86.2 & 86.5 & 86.0 & 84 & 84.0 & 82.1 \\
\hline & 94.8 & 95.3 & 95.0 & 95.0 & 95.3 & 95.0 & 93.9 & 94.8 & 95.3 & 97.0 & & 95.9 & 94.2 & 96.1 & 95.09 & 95.0 & 87.3 & 87.1 & 86.8 & 86.8 & 86.2 & 8 & 82.4 \\
\hline & 94.8 & 94.8 & 94.5 & 94.5 & 94.8 & 94.5 & 93.4 & 94.2 & 94.8 & 94.2 & 95.9 & & 96.7 & 94.2 & 94.5 & 94.58 & 87.3 & 86.2 & 87.3 & 86.5 & 85.4 & 84.6 & 82.4 \\
\hline 13.81 & 94.2 & 94.2 & 93.9 & 93.9 & 93.7 & 93.4 & 92.8 & 93.7 & 94.5 & 92.3 & 94.2 & 96.7 & & & 94.5 & 93.9 & 85.4 & 84.3 & 86.5 & 84.3 & 84 & 82.4 & 82.4 \\
\hline & 94.5 & 95.0 & 94.8 & 94.8 & 94.5 & 94.8 & 94.2 & 95.0 & 95.9 & 94.5 & 96.1 & 94.2 & 94.2 & & 94.8 & 94.8 & 87.6 & 86.8 & 86.0 & 86.0 & 84.8 & 83.7 & 82.1 \\
\hline & 98.6 & 99.2 & 97.2 & 98.3 & 97.2 & 97.0 & 95.9 & 97.8 & 95.9 & 93.1 & 95.0 & 94.5 & 94.5 & 94.8 & & 97.2 & 87.6 & 85.7 & 85.7 & 86.0 & 84.8 & 82.9 & 82.9 \\
\hline & 97.5 & 98.1 & 100.0 & 98.3 & 97.2 & 97.0 & 95.3 & 97.2 & 95.0 & 92.8 & 95.0 & 94.5 & 93.9 & 94.8 & 97.2 & & 88.7 & 87.3 & 87.3 & 87.3 & 86.0 & 3.5 & 82.9 \\
\hline & 88.4 & 87.3 & 88.7 & 88.2 & 88.2 & 89.0 & 87.3 & 88.2 & 87.9 & 86.5 & 87.3 & 87.3 & 85.4 & 87.6 & 87.6 & 88.7 & & 94.8 & 82.6 & 83.5 & 83.2 & 82.1 & 80.4 \\
\hline 18. W & 86.2 & 86.0 & 87.3 & 86.2 & 866.5 & 87.1 & 86.0 & 86.8 & 86.0 & 86.2 & 87.1 & 86.2 & 84.3 & 86.8 & 85.7 & 87.3 & 94.8 & & 84.6 & 84.0 & 83.7 & 82.6 & 81.3 \\
\hline & 86 & 86 & 87.3 & 86.8 & 86.2 & 86.2 & 84.6 & 86.2 & 86.5 & 86.5 & 86.8 & 87.3 & 86.5 & 86.0 & 85.7 & 87.38 & 82.6 & 84.6 & & 87.3 & 89.5 & 84.6 & 84.3 \\
\hline & 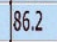 & 86.8 & 87.3 & a & 87.1 & 87.3 & 87.1 & 86.8 & 86.0 & 86.0 & 86.8 & 86.5 & 84.3 & 86.0 & 86.0 & 87.3 & 83.5 & 84.0 & 87.3 & & 86.8 & 87.1 & 35.7 \\
\hline I Wrk & 85.4 & 85.1 & 86.0 & 85.4 & 85.1 & 85.7 & 84.8 & 84.3 & 85.1 & 84.6 & 86.2 & 85.4 & 84.8 & 84.8 & 84.8 & 86.08 & 83.2 & 83.7 & 89.5 & 86.8 & & 84.3 & 84.3 \\
\hline & 82 & & 83.5 & & 82.6 & 82.9 & 83.7 & 82.9 & 83.2 & 84.0 & 84.8 & 84.6 & 82.4 & $0.1 \%$ & 82.9 & 83.58 & 82.1 & 82.6 & 84.6 & 87.1 & 84.3 & & 36.0 \\
\hline HTI & 82. & & 9 & & 82.6 & 81.8 & 83.7 & 81.8 & 81.8 & 1 & & 82 & & & 82.9 & & 80.4 & 81.3 & 84.3 & 55.7 & 84.3 & 86.0 & \\
\hline
\end{tabular}

Fig. 3. Detailed nucleic acid identity and similarity rates for CaKV/TR/2018 (Turkish) and other kobuviruses marked by red color in SDT (Fig. 2)

the pink and green geographical zones. Pig, sheep, and cattle strains were light green and blue with $\mathrm{CaKV} /$ $\mathrm{TR} / 2018$. The most significant divergences within the group were for bovine kobuviruses, klassevirus, gray-backed thrush turdivirus, and chicken sicinivirus, as shown by dark blue (Fig. 2). The nucleotide identities and divergences of 23 different isolates analyzed in Figure 3 were selected according to the color scale in Figure 2 and found close to our field strain. The most significant difference according to the nucleotide identities in Figure 3 was found for CaKV/ TR/2018, with $82.1 \%$ homology with KY855436.1/ Marmot-kobuvirus/HT9 strain. Apart from the US strain AN211D/JN387133/CaKV/USA, the highest amino acid sequence identity of CaKV/TR/2018 was $95.9 \%$ with CoKV/61c/2012/IT_KC693046.1/Italy strain. Our strain also showed high identities (95\%) with HRB-H6/ KT210424.1/CaKV/China and 12D049/KF924623.1/ $\mathrm{CaKV} /$ South Korea strains. CaKV/TR/2018 likewise demonstrated high similarities with AB861494.1/Kobuvirus/sewage/dog/Aichi/Y12/2004/Japan, KT210414.1/ CaKV/HRB-D9/China, LC147656.1/CaKV/ Tokyo1173-2012-JP, MF062167/CaKV/SMCQ-M19/ China, and AB861494.1/Kobuvirus sewage/Aichi/Y12/ Japan strains, which had approximately $0.5 \%$ diversity and $94.8 \%$ similarity at the nucleotide level. CaKV strains from China and Japan had the closest similarities, followed by the other Japanese (LC147655.1/CaKV/ Ca-Gifu0226/Japan) and Chinese strains (JQ911763.1/ $\mathrm{CaKV} / \mathrm{CH}-1 / \mathrm{China}$ ), with $94.5 \%$ sequence identity. Two Tanzania CaKV strains (KM068048.1/CaKV/DD2/ Tanzania, KM068051.1/CaKV/B103/Tanzania) and a UK CaKV strain (KC161964.1/CaKV/UK003/UK) both had a sequence identity of $94.2 \%$ with $\mathrm{CaKV} /$
TR/2018. Kobuviruses from cat species (KJ958930.1/ Feline-kobuvirus/12D240 and MF598159.1/Feline-kobuvirus/WHJ-1) shared an identity of $87.6 \%$ and $86.8 \%$, respectively, with the CaKV strain, characterized in this study at the nucleotide level. The untreated sewage (JQ898342.1/Kobuvirus-sewage/KoV-SewKTM/ Nepal) and mouse (MF175074.1/Murine-kobuvirus/ MKV1/NYC) strains shared a lower homology than CaKVs at $86.0 \%$. The other Kobuvirus strains from different species of Muridae (KJ950909.1/Norwayrat-kobuvirus/NrKoV-1/NYC, JF755427.1/Mousekobuvirus/M-5/USA, and KY855436.1/Marmot-kobuvirus/HT9) also showed low nucleotide homologies with $\mathrm{CaKV} / \mathrm{TR} / 2018$, at with $84.8 \%, 83.7 \%$, and $82.1 \%$, respectively, as shown in Fig. 2.

While CaKVs has been detected in both healthy and diarrheic dogs in several countries, such as Italy, South Korea, Japan, US, China, and Tanzania, there is no information about the infection in Turkey $(2,5,8$, 10). In many studies, CaKVs are detected as common agents of dogs while its presence in dogs is mostly coinfection with other dog pathogens, such as CPV-2 or $\mathrm{CCoV}$. Single infection detections have also been made $(5,8,12)$.

In the present study, the presence of CaKV in the rectal swab of a dog was determined using RT-PCR while the sequencing and molecular characterization study identified the field strain as CaKV. Regarding its phylogenetic relationship with 48 kobuviruses from 7 different species, aichiviruses, klassevirus, turdivirus, and siciniviruses were specified as the outgroups.

The full genome sequence analysis showed that dog kobuviruses are closest to murine kobuvirus and diarrhea-causing human aichivirus, and classified in 
Aichivirus A group $(10,15)$. In our study, canine kobuviruses in the phylogenetic tree had higher homology with feline and murine kobuviruses whereas they were distant from human aichivirus, though still with about $85 \%$ similarity. An earlier study revealed that the complete genome of a CaKV strain from Korea was located in a lineage that was also distinct from AiV (12). Carmona-Vicente et al. (2) obtained other data supporting this finding in that sequence analysis indicated $\mathrm{CaKV}$ was distinct from human aichivirus.

Chung et al. (4) detected CaKV in cat stool samples and found that feline kobuviruses were most closely related to canine kobuvirus. Thus, cross-species transmission may play a role in the close relationship we observe with cat kobuviruses.

In our phylogenetic tree, although GQ927711 and FJ225407 Aichi viruses are both human-origin strains, GQ927711 Aichivirus was placed as a separate branch while FJ225407Aichivirus was located as a sister branch in the sicinivirus cluster. Porcine kobuviruses formed a separate group while caprine kobuvirus formed a separate branch within the porcine kobuvirus group. All bovine kobuviruses placed in the same cluster as sheep kobuviruses while KF006986 Ferret Kobuvirus and one of the bovine strains were also located within this group. From their full genome analysis of ferret kobovirus, Smits et al. (17) reported that kobuvirus from rectal swab was more closely associated with bovine and ovine kobuviruses in the Aichivirus B group. Our study also supports this finding.

Unlike in other species, the murine kobuviruses used in this phylogenetic tree did not show high percentage similarity on their own and they demonstrated a similar nucleotide homology as they showed to canine and feline viruses. Mouse kobuviruses showed a homology between $82 \%$ and $86 \%$ with our local field strain.

The dog is the world's most widely-fed and the closest pet; hence, as companion animals, their diseases are also important for human health. Since kobuviruses have been detected in humans, in further studies, probable mutations and similarities of CaKV should also be considered from the point of view of viral evolution. The present study showed that CaKV was detected in the rectal swab of a dog with diarrhea in Turkey. Sequence analysis indicated that canine kobuviruses used from the genebank in this study are closely associated with each other and with our strain $\mathrm{CaKV} /$ TR/2018 by compromising a separate cluster. Although they are RNA viruses, no significant genetic variation was observed among the CaKVs whose relationships were investigated. However, the investigated area was a region of only $500 \mathrm{bp}$ so future full genome analyses will provide more accurate results. We reported here the first detection and phylogenetic analysis of canine kobuviruses in Turkey. Further epidemiological studies are required to characterize kobuviruses as a viral pathogen in the gastrointestinal infections of dogs and other mammalians in Turkey. Regarding dogs, it is es- sential to determine how frequently kobuviruses are found compared to other viruses (rota, corona, parvo, etc.) that circulate in gastrointestinal flora and to investigate them in diagnostic panels. Finally, obtaining these strains may help provide the infrastructure for future diagnostic kits and vaccines by determining CaKV's infection mechanism.

\section{References}

1. Amimo J. O., Okoth E., Junga J. O., Ogara W. O., Njahira M. N., Wang Q., Vlasova A. N., Saif L. J., Djikeng A.: Molecular detection and genetic characterization of kobuviruses and astroviruses in asymptomatic local pigs in East Africa. Arch. Virol. 2014, 159, 1313-1319.

2. Carmona-Vicente N., Buesa J., Brown P. A., Merga J. Y., Darby A. C., Stavisky J., Sadler R. M., Gaskell S., Dawson A. D.: Phylogeny and prevalence of kobuviruses in dogs and cats in the UK. Vet. Microbiol. 2013, 164, 246-252.

3. Chomczynski P., Sacchi N.: Single-step method of RNA isolation by acid guanidinium thiocyanate-phenol-chloroform extraction. Anal. Biochem. 1987, 162, 156-159.

4. Chung J. Y., Kim S. H., Kim Y. H., Lee M. H., Lee K. K., Oem J. K.: Detection and genetic characterization of feline kobuviruses. Virus Genes 2013, 47, 559-562.

5. Di Martino B., Di Felice E., Ceci C., Di Profio F., Marsilio F.: Canine kobuviruses in diarrhoeic dogs in Italy. Vet. Microbiol. 2013, 166, 246-249.

6. Greninger A. L., Knudsen G. M., Betegon M., Burlingame A. L., Derisi J. L. The 3 A protein from multiple picornaviruses utilizes the golgi adaptor protein ACBD3 to recruit PI4KIII $\beta$. J. Virol. 2012, 86, 3605-3616.

7. Kapoor A., Simmonds P., Dubovi E. J., Qaisar N., Henriquez J. A., Medina J., Shelly S., Lipkin W. I.: Characterization of a canine homolog of human Aichivirus. J. Virol. 2011, 85, 11520-11525.

8. Kong N., Zuo Y., Wang Z., Yu H., Zhou E. M., Shan T., Tong G.: Molecular characterization of new described kobuvirus in dogs with diarrhea in China. Springerplus 2016, 5, 2047

9. Lee M. H., Jeoung H. Y., Lim J. A., Song J. Y., Song D. S., An D. J.: Kobuvirus in South Korean black goats. Virus Genes 2012, 45, 186-189.

10. Li L., Pesavento P. A., Shan T., Leutenegger C. M., Wang C., Delwart E. Viruses in diarrhoeic dogs include novel kobuviruses and sapoviruses. J. Gen. Virol. 2011, 92, 2534.

11. Muhire B. M., Varsani A., Martin D. P.: SDT: a virus classification tool based on pairwise sequence alignment and identity calculation. PlOS One 2014, 9 , e108277.

12. Oem J. K., Choi J. W., Lee M. H., Lee K. K., Choi K. S.: Canine kobuvirus infections in Korean dogs. Arch. Virol. 2014, 159, 2751-2755.

13. Oh D. Y., Silva P. A., Hauroeder B., Diedrich S., Cardoso D. D., Schreier E.: Molecular characterization of the first Aichi viruses isolated in Europe and in South America. Arch. Virol. 2006, 151, 1199-1206.

14. Pankovics P., Boros Á., Kiss T., Reuter G.: Identification and complete genome analysis of kobuvirus in faecal samples of European roller (Coracias garrulus): for the first time in a bird. Arch. Virol. 2015, 160, 345-351.

15. Phan T., Kapusinszky B., Wang C., Rose P. K., Lipton H. L., Delwart E.: The fecal viral flora of wild rodents. PLOS Pathog. 2011, 7, p. e1002218.

16. Reuter G., Boldizsár Á., Pankovics P.: Complete nucleotide and amino acid sequences and genetic organization of porcine kobuvirus, a member of a new species in the genus Kobuvirus, family Picornaviridae. Arch. Virol. 2009, $154,101$.

17. Smits S. L., Raj V. S., Oduber M. D., Schapendonk C. M., Bodewes R., Provacia L., Stittelaar K. J., Osterhaus A. D., Haagmans B. L.: Metagenomic analysis of the ferret fecal viral flora. PLOS One 2013, 8, p. e71595.

18. Tamura K., Stecher G., Peterson D., Filipski A., Kumar S.: MEGA6: Molecular Evolutionary Genetics Analysis version 6.0. Mol. Biol. Evol. 2013, 30, 2725 -2729 .

19. Yamashita T., Shinichi K., Kcnji S., Shuji N., Shunzo C., Yuichi I., Shin I.: Isolation of cytopathic small round viruses with BS-C-1 cells from patients with gastroenteritis. J. Infect. Dis. 1991, 164, 954-957.

20. Yamashita T., Ito M., Kabashima Y., Tsuzuki H., Fujiura A., Sakae K.: Isolation and characterization of a new species of kobuvirus associated with cattle. J. Gen. Virol. 2003, 84, 3069-3077.

Corresponding author: Dr. Zeynep Akkutay-Yoldar, DVM, PhD, Ankara University, Faculty of Veterinary Medicine, Department of Virology, 06110, Ankara, Turkey; e-mail: akkutay@ankara.edu.tr 